\title{
Rotation and Massive Close Binary Evolution
}

\author{
Norbert Langer ${ }^{1}$, Matteo Cantiello ${ }^{1}$, Sung-Chul Yoon ${ }^{2}$, Ian Hunter ${ }^{3}$, \\ Ines Brott ${ }^{1}$, Danny Lennon ${ }^{4}$, Selma de Mink ${ }^{1} \&$ Marcel Verheijdt ${ }^{1}$ \\ ${ }^{1}$ Astronomical Institute, Utrecht University, The Netherlands \\ ${ }^{2}$ University of California, Santa Cruz, USA \\ ${ }^{3}$ The Queen's University of Belfast, Northern Ireland, UK \\ ${ }^{4}$ Isaac Newton Group, Santa Cruz de La Palma, Canary Islands, Spain
}

\begin{abstract}
We review the role of rotation in massive close binary systems. Rotation has been advocated as an essential ingredient in massive single star models. However, rotation clearly is most important in massive binaries where one star accretes matter from a close companion, as the resulting spin-up drives the accretor towards critical rotation. Here, we explore our understanding of this process, and its observable consequences. When accounting for these consequences, the question remains whether rotational effects in massive single stars are still needed to explain the observations.
\end{abstract}

Keywords. stars: early-type - stars: fundamental parameters - stars: mass loss - stars: rotation - stars: binaries: close

\section{Why look at rotation?}

Rotation has been identified as an important physics ingredient which needs to be considered to understand the evolution of massive star (e.g., Heger, Langer \& Woosley 2000; Meynet \& Maeder 2000). It is thought to gives rise to physical effects inside stars which cause observable quantities to change, and may even radically alter the evolutionary path of the stars. A drastic example is the occurrence of chemically homogeneous evolution, which may provide a progenitor path towards long gamma-ray bursts (Yoon \& Langer 2005, Yoon et al. 2006, Woosley \& Heger 2006).

One of the most relevant prediction of massive star models with rotation is that rotationally triggered internal transport processes are capable to bring nuclear processed material, most notably nitrogen, from the convective core of massive main sequence stars into their radiative envelope. For fast enough rotation, fresh nitrogen thus appears at the surface of the star, and becomes continuously more enriched as function of time during core hydrogen burning.

Numerous incidental evidences have been collected from observations which are in support of this picture (cf. Maeder \& Meynet 2000, and references therein). However, while many observations refer to stars in their post-main sequence stages, abundance analyses of main sequence stars have mostly been restricted to apparent slow rotators and to rather small groups of stars. A major step forward in comparing massive star models and observations is provided by the FLAMES Survey of Massive Stars, which encompassed many hundred $\mathrm{O}$ and early B main sequence stars in the Galaxy and the Magellanic Clouds (Evans et al. 2005). The B star sample of this survey was analyzed in a way which allowed for the first time to obtain quantitative constrains on the nitrogen enhancement also in a large number of rapid rotators (Hunter et al. 2008, Fig. 1). Surprisingly, Hunter et al. could not unambiguously conclude that the effects of rotation 


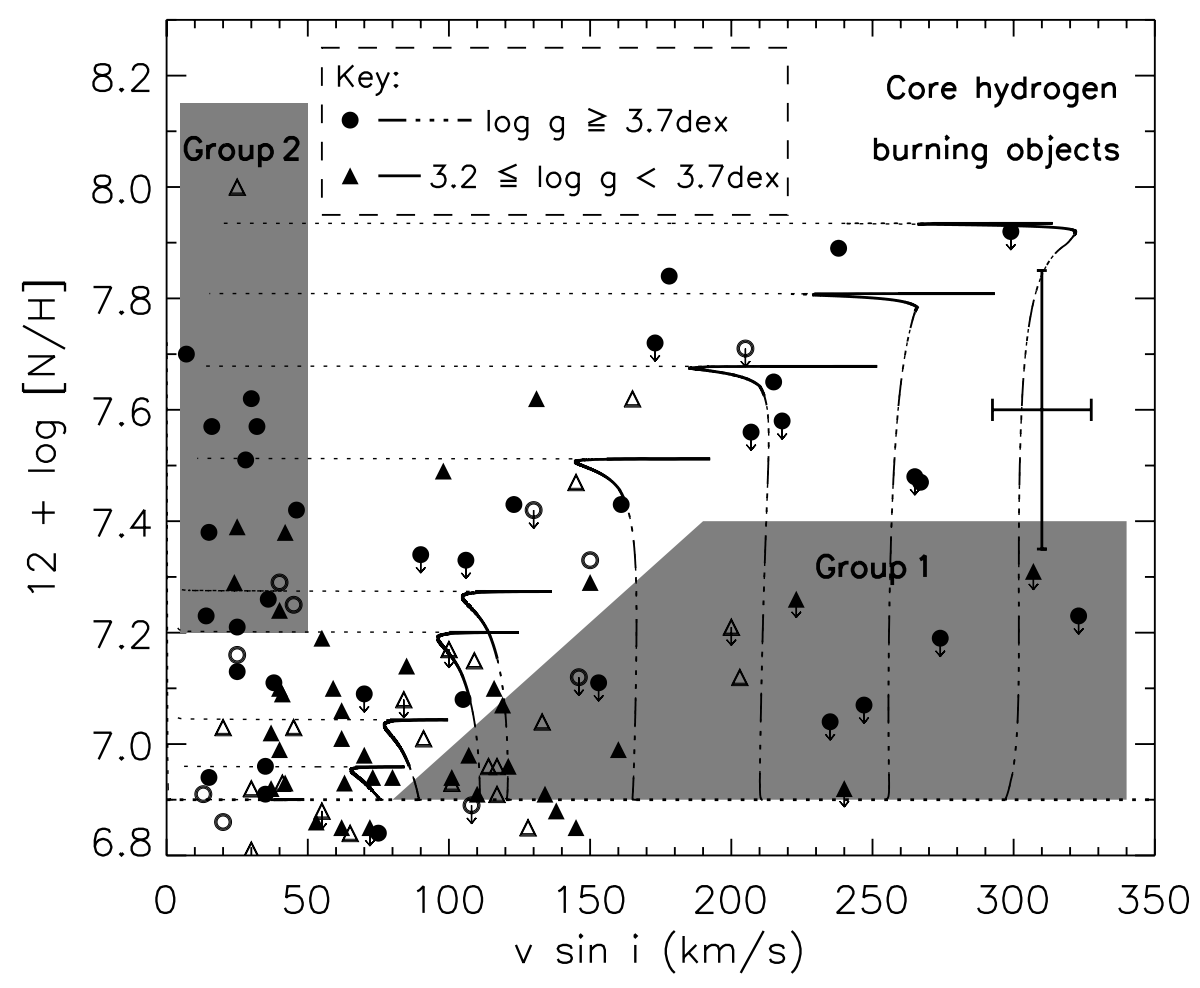

Figure 1. Nitrogen abundance $(12+\log [\mathrm{N} / \mathrm{H}])$ against the projected rotational velocity $(v \sin i)$ for core hydrogen burning objects in two LMC fields centered on N11 and NGC2004, according to Hunter et al. (2008). Open symbols: radial velocity variables; downward arrows: abundance upper limits; lowest dotted line: LMC baseline nitrogen abundance. The mean uncertainty in the nitrogen abundance is 0.25 dex while that in $v \sin i$ is $10 \%$. The bulk of the stars occupy a region at low $v \sin i$ and show little or modest nitrogen enrichment. The stellar evolution tracks are computed for an initial mass of $13 \mathrm{M}_{\odot}$ corresponding to the average mass of the sample stars, and their rotational velocity has been multiplied by $\pi / 4$ to account for random inclinations. The surface gravity is used as indicator of the evolutionary status and the objects (see legend) and tracks have been split to indicate younger and older core hydrogen burning stars, respectively. Gray shading highlights two groups of stars which remain unexplained by the stellar evolution tracks.

are observed as expected. They rather found two groups of rapid rotators, one with significant enrichment and one without (the latter being dubbed Group 1 in Fig. 1). Both groups contain stars which are close to core hydrogen exhaustion, as indicated by their low surface gravity.

Hunter et al. (2008) gave two possible ways of interpretation. The one which saves the current picture of rotational mixing is that the enriched fast rotators in the FLAMES sample are indeed single stars, while the non-enriched fast rotators have a peculiar binary history. An observing campaign is underway to test the hypothesis that these latter stars are indeed all binaries - for which in the current FLAMES data there is no clear evidence. Alternatively, the results of Hunter et al. (2008) could imply that rotational mixing is not efficient, and that the enriched fast rotators are all spun-up accretion stars in binaries. The worry that the latter might be true is strengthened by the finding of yet another 
discrete group of massive main sequence stars by Hunter et al. (2008; named Group 2 in Fig. 1), namely intrinsically slowly rotating stars with a strong nitrogen enhancement. While this group of stars clearly needs an alternative explanation, it appears likely that previous reports of nitrogen enrichment in massive main sequence stars which served as support for rotational mixing picked up stars comparable to those in Group 2, as they were limited to low projected rotational velocities.

In the following, we discuss which possibilities are supported by current models of massive close binaries. As mentioned above, it appears impossible to understand the nitrogen pattern in fast rotators without invoking close binaries. One may actually wonder whether even all fast rotators could be produced by close binary effects.

\section{Required physics}

Massive close binary evolution is modeled by various groups (e.g., Podsiadlowski et al. 1992, Wellstein \& Langer 1999, Belczynski et al. 2002, Vanbeveren et al. 2007, Vazquez et al. 2007, Eldridge et al. 2008). However, in order to predict the surface nitrogen abundances and the rotational velocities of binary components, a rather large amount of physical effects needs to be considered in binary evolution models.

It is desirable to include, in such binary models, the physics of rotation as it is currently used in models of rotating massive single stars. The reason is that in close binaries, even rather small amounts of matter transferred during Roche-lobe overflow spins up the mass gainer to extreme rotation rates (Packet 1981). Therefore, if rotational mixing is real, it might have the strongest effects in binary systems.

Angular momentum transport by internal magnetic fields is one ingredient in single star models which appears to be indispensable as well. Heger et al. (2005) showed that without this effect, young neutron stars are predicted to spin too rapidly. Suijs et al. (2008) showed that magnetic transport is also required to prevent too fast rotation in white dwarfs (Fig. 2).

For binary evolution models, there are two more pieces of physics which need to be included, which both relate to angular momentum exchange between the components of a binary system. Mass transfer within the Roche approximation is commonly applied in binary evolution calculations, but the corresponding angular momentum transfer is mostly neglected. The latter is crucial to model the spin-up of the accretion star, and thus to represent the most rapidly rotating stars at all. Spin-orbit coupling through tides is the other unmissable ingredient in close binary models, as it can lead to significant spin-down (mostly!) or spin-up (rarely) in massive binaries with periods below $10 \ldots 20 \mathrm{~d}$.

Wellstein (2001) and Petrovic et al. (2005a, 2005b; see also Detmers et al. 2008) have produced a binary code which includes all the required physics. While only few binary evolution models have been computed with this code so far, these models may help to answer a few of the questions raised above. Some of their properties are discussed below.

\section{Luminosity and effective temperature}

We want to briefly discuss two important effects of binarity on the distribution of stars in the HR diagram, in particular concerning the mass gainer, which will be the more prominent star of the two after a mass transfer event.

Fig. 3 shows that mass gainers become more luminous the more mass they gain. However, if the accreted amount of matter is large, and if it occurs late enough during the core hydrogen burning evolution of the mass gainer, its rejuvenation, i.e. in particular the growth of its convective core to adapt to the increased stellar mass, might be avoided 


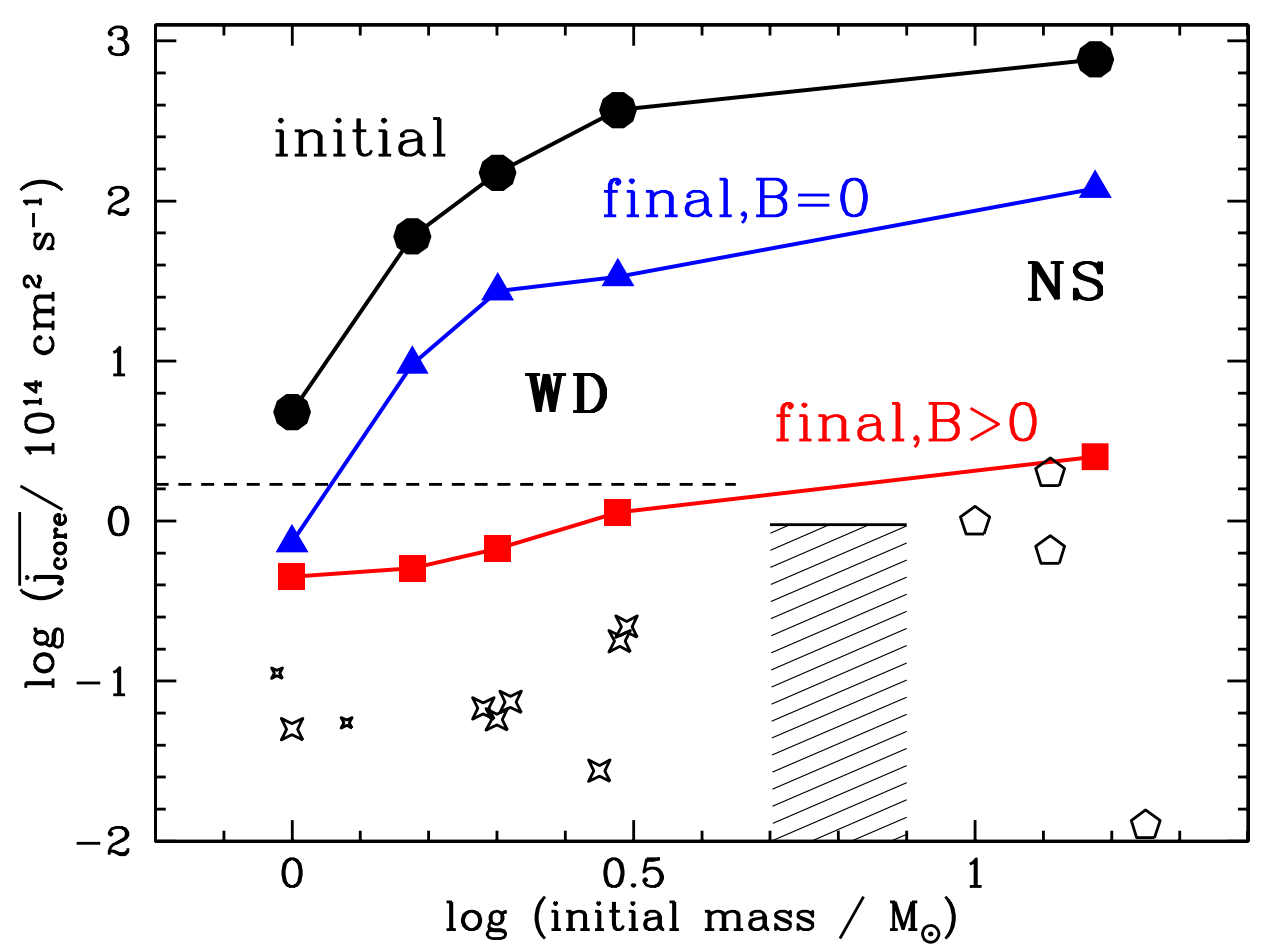

Figure 2. Average initial (upper line) and final core specific angular momentum of $1 \ldots 3 \mathrm{M}_{\odot}$ stars according to Suijs et al. (2008), and $15 \mathrm{M}_{\odot}$ stars according to Heger et al. (2005). Filled triangles corresponds to the final models of non-magnetic sequences, and filled squares to the final models of magnetic sequences. The dashed horizontal line indicates the spectroscopic upper limit on the white dwarf spins obtained by Berger et al. (2005). Star symbols represent astroseismic measurements from ZZ Ceti stars (Bradley 1998, 2001; Dolez 2006; Handler 2001, Handler et al. 2002, Kepler et al. 1995, Kleinmann et al. 1998, Winget et al. 1994), where smaller symbols correspond to less certain measurements. The hatched area is populated by magnetic white dwarfs (Ferrario \& Wickramasinghe 2005; Brinkworth et al. 2007). The three open pentagons correspond to the youngest Galactic neutron stars (Heger et al. 2005). The lowest pentagon is thought to roughly correspond to magnetars (Camilo et al. 2007).

(Braun \& Langer 1995). In this case, core helium burning may take place very close to the main sequence, i.e., helium burning stars may be mistaken for main sequence stars. In this respect, we point out that Hunter et al. (2008) interpreted the sharp drop of the projected rotational as function of surface gravity as signaling the cool end of the main sequence band.

The increased luminosity of the mass gainers, which is also clearly visible in the evolutionary tracks shown in Fig. 4, may cause them to appear as blue stragglers in samples of stars with similar age (cf., Pols \& Marinus 1994). If then a sample of stars is defined through a visual magnitude cut-off, which might favor evolved main sequence stars near the turn-off, it is conceivable that mass gainers constitute a significant fraction of the whole sample.

\section{Distribution of rotational velocities}

In order to model the distribution of stars in Fig. 1, one requires a distribution function for the initial rotational velocity of single stars (IRF). However, what can be measured is 


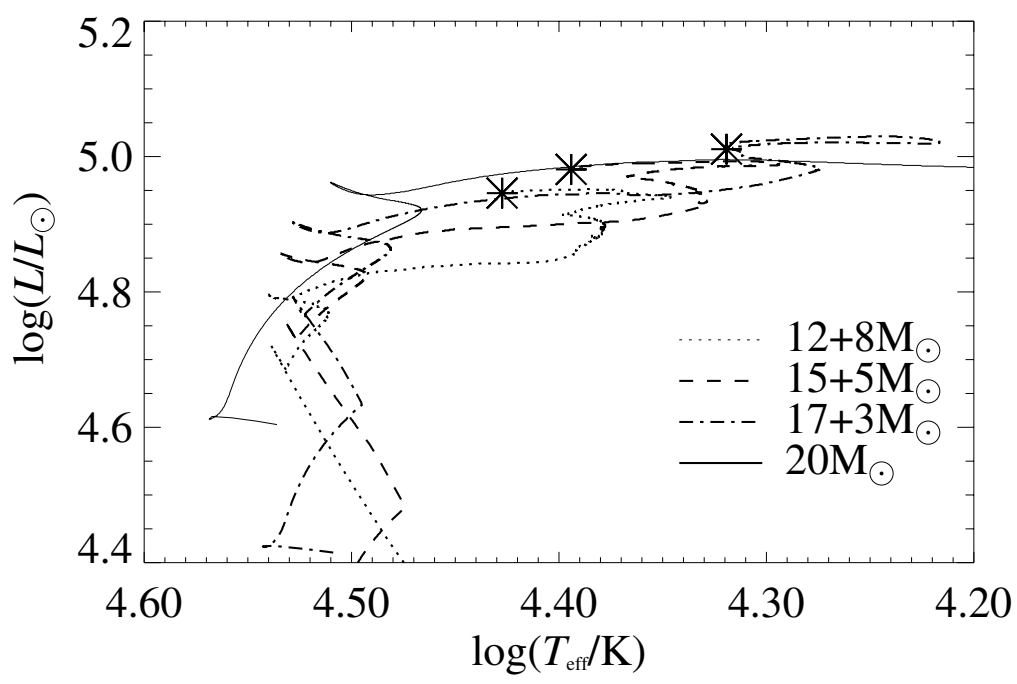

Figure 3. Tracks in the HR diagram for stars with a total mass of $20 \mathrm{M}_{\odot}$ after accretion at a central helium mass fraction of $Y=0.7$, starting at $12 \mathrm{M}_{\odot}, 15 \mathrm{M}_{\odot}$, and $17 \mathrm{M}_{\odot}$ (see legend), compared to the track of a $20 \mathrm{M}_{\odot}$ single star (Braun \& Langer 1995). While the single star evolves to the red supergiant stage immediately after core hydrogen exhaustion, the accreting stars, which do not rejuvenate, remain blue supergiants throughout core helium burning. Their pre-supernova position is indicated be an asterisk.

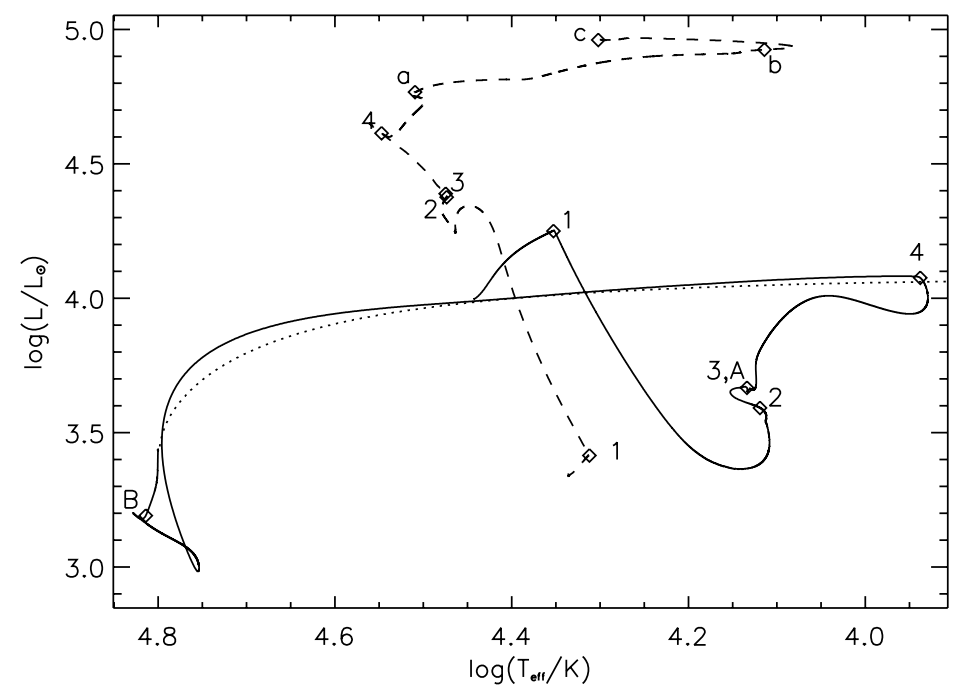

Figure 4. Evolutionary tracks of the primary (solid and dotted line) and secondary star (dashed line) of the case A binary system No. 31 of Wellstein et al. (2001; initial masses are $12 \mathrm{M}_{\odot}$ and $7.5 \mathrm{M}_{\odot}$, the initial period is $2.5 \mathrm{~d}$ ) in the HR diagram. Beginning and end of the mass transfer phases are marked with numbers; 1: begin of Case A, 2: end of Case A, 3: begin of Case $\mathrm{AB}, 4$ : end of Case $\mathrm{AB}$. The labels $\mathrm{A} /$ a designate the end of central hydrogen burning of the primary/secondary, $\mathrm{B} / \mathrm{b}$ the end of central helium burning of the primary/secondary, and $\mathrm{c}$ the point of the supernova explosion of the secondary. In this system, the secondary star ends its evolution first. The time of its supernova explosion marks the end of the solid line in the track of the primary. The further evolution of the primary is shown as dotted line. During this phase, it is treated as a single star since the system is likely broken up due to the secondary's explosion. 


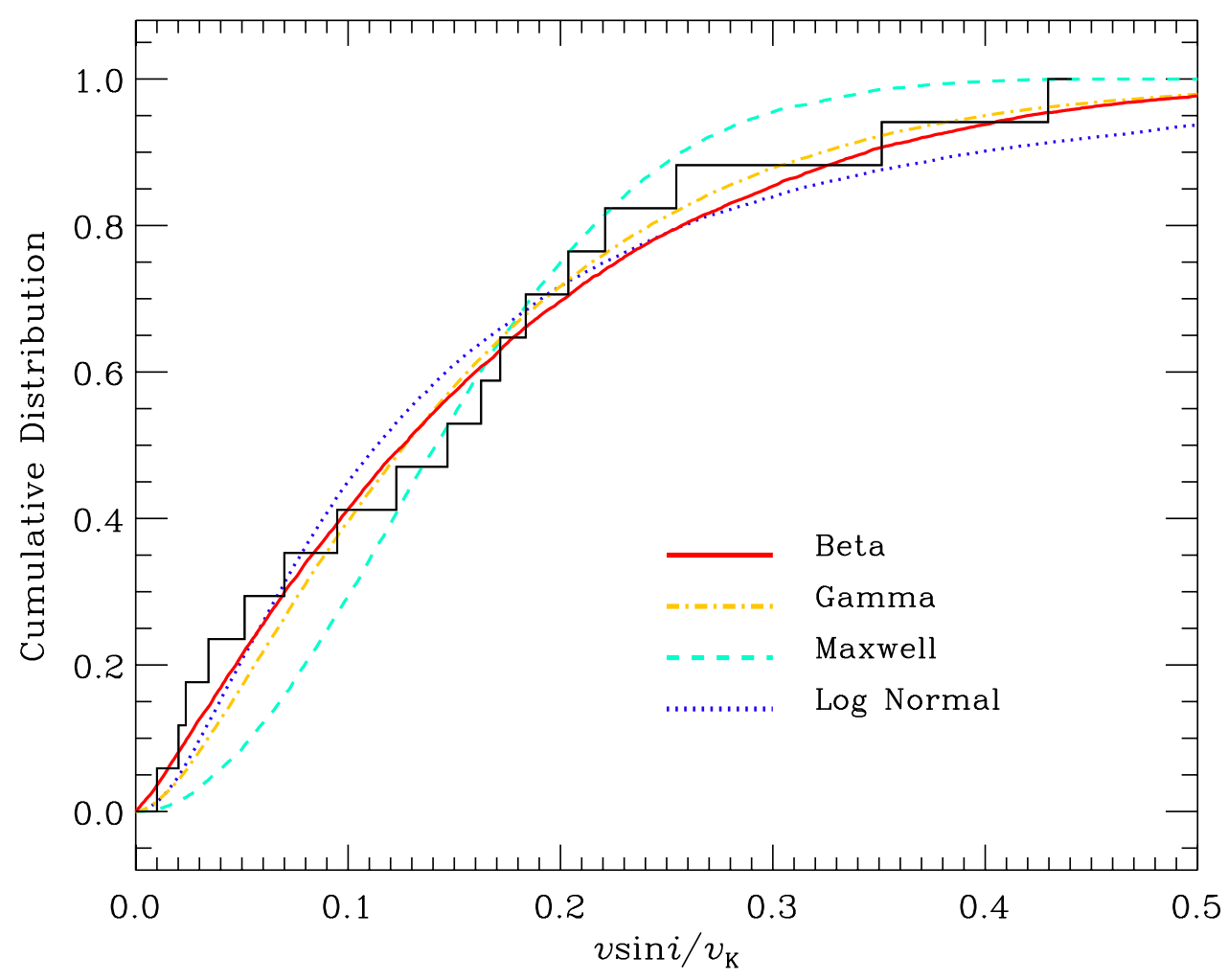

Figure 5. Cumulative distribution of the fraction of the Keplerian value of the observed rotational velocity (i.e., $v \sin i$ ) of unevolved young stars in NGC 346 in the Small Magellanic Cloud, according to Yoon et al. (2006). The data (step function) is from Mokiem et al. (2006). The dotted-dashed, solid, and dashed lines are the best fits of synthesized distribution functions using three different distribution laws: beta, gamma and Maxwellian, respectively. Here it is assumed that the stellar rotation axes are randomly oriented.

only the present-day distribution of rotational velocities (PRF). Fig. 5 gives an example derived within the FLAMES survey, which is the PRF of the $\mathrm{O}$ stars in an SMC field centered on NGC 346 (Mokiem et al. 2006). The Magellanic Clouds are good study grounds for this, since at least spin-down of single stars by radiation driven winds can be neglected for all except the very most massive main sequence stars. Worrisome about Fig. 5 is that all but the fastest three rotators have rotation rates of less than a quarter of critical rotation, while two of the three fast rotators appear to be runaway stars. We thus ask the question: could all rapid rotators be binary products?

The top panels of Figs. 6 and 7 show the evolution of the rotational velocity of mass gainers for two different binary evolution models (Wellstein 2001). The mass gainer will be the dominantly visible star after the first accretion event, and the mass loser may be hard to notice at all or even be ejected through its supernova explosion. These figures show that very rapid rotators are produced, which are long-lived main sequence stars. Due to the blue straggler effect, they may dominate certain parts of the HR-diagram (cf. Section 3).

Fig. 8 shows the range of contact-free evolution in the initial period versus initial mass ratio diagram for primary star masses of relevance here. Mass transfer and spin-up is expected everywhere within the contact-free regime (Wellstein et al. 2001). However, in 


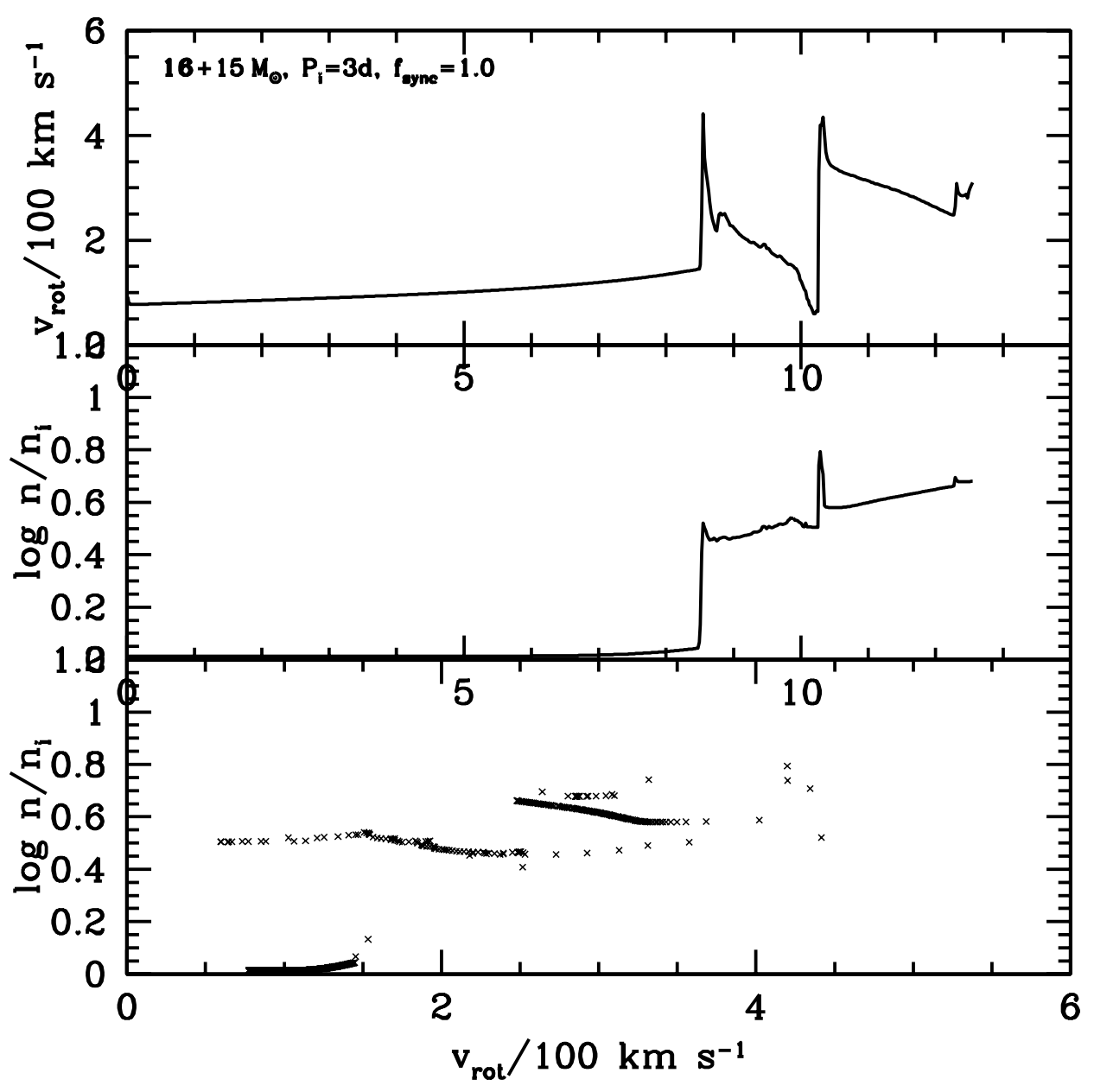

Figure 6. Equatorial rotational velocity (upper panel) and surface nitrogen mass fraction relative to the initial value (middle panel) as function of time, for a the mass gainer in a solar metallicity $16 \mathrm{M}_{\odot}+15 \mathrm{M}_{\odot}$ binary with an initial period of 3 days. Time is given in Myr for the upper two panels. The computations include the physics of rotation for both components as in Heger et al. (2000), and Spin-Orbit coupling as in Detmers et al. (2008) with the nominal coupling parameter $f_{\text {sync }}=1$, and rotationally enhanced stellar wind mass loss (Langer 1998). Internal magnetic fields are not included. The bottom panel shows the evolution of the mass gainer in the nitrogen enhancement versus rotational velocity diagram, where each data point represents a duration of $20000 \mathrm{yr}$. The spin-down of the star after the first accretion event $(t=8.5 \ldots 10 \mathrm{Myr})$ is mostly due to tidal effects. This example shows that massive close binaries can produce rotating nitrogen rich stars which are rapidly rotating, but also such which are slowly rotating.

most regions outside of this, both stars in the binary are expected to merge as a result of their interaction (see also Podsiadlowski et al. 1992). While the details of the merger process are difficult to predict, the merger product will be an extreme rotator due to the enormous surplus of angular momentum. Merger stars will only be observed as single stars.

We see that due to mass transfer and merging, a stellar population with few or no rapid rotators initially may build up a certain number of rapidly rotating core hydrogen 


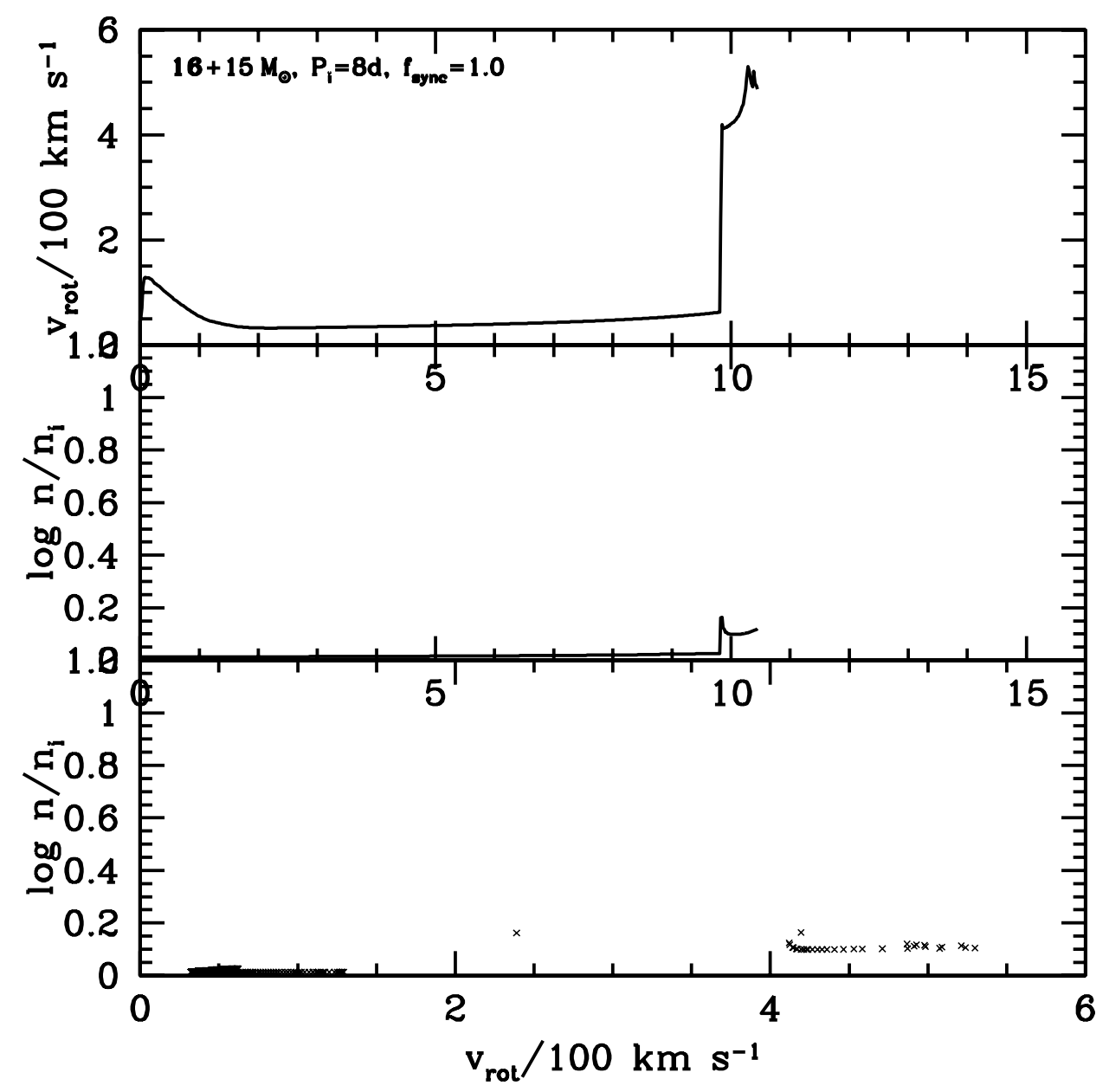

Figure 7. As Fig. 6, but for a $16 \mathrm{M}_{\odot}+15 \mathrm{M}_{\odot}$ binary with an initial period of 8 days, which makes it a Case B system. Due to the larger initial period, the mass transfer is very non-conservative. Enough mass is accreted to spin-up the mass gainer, but not enough to create a large nitrogen surface enhancement. This example shows that massive close binaries can produce rapidly rotating, evolved main sequence stars which are not strongly nitrogen-enriched. Note that the calculations stops about $1 \mathrm{Myr}$ after the mass transfer due to numerical difficulties. Possibly, the mass gainer would become nitrogen-enhanced later on due to rotational mixing.

burning stars. Some of them may slow down again due to their close companion (cf. Fig. 6, top panel), but many will not. It will be an important task for the near future to put quantitative limits on the relative number of those stars in stellar populations.

\section{Nitrogen enrichment}

The surface nitrogen abundance of mass gainers in close binary systems can be affected in two major ways. First of all, the matter which is accreted from the companion is often nitrogen-rich, as it stems from deep layers of the initial primary. While the transferred matter may be very nitrogen-rich, with values close to CNO-equilibrium being reached toward the end of the mass transfer, the enrichment on the mass gainer remains limited 


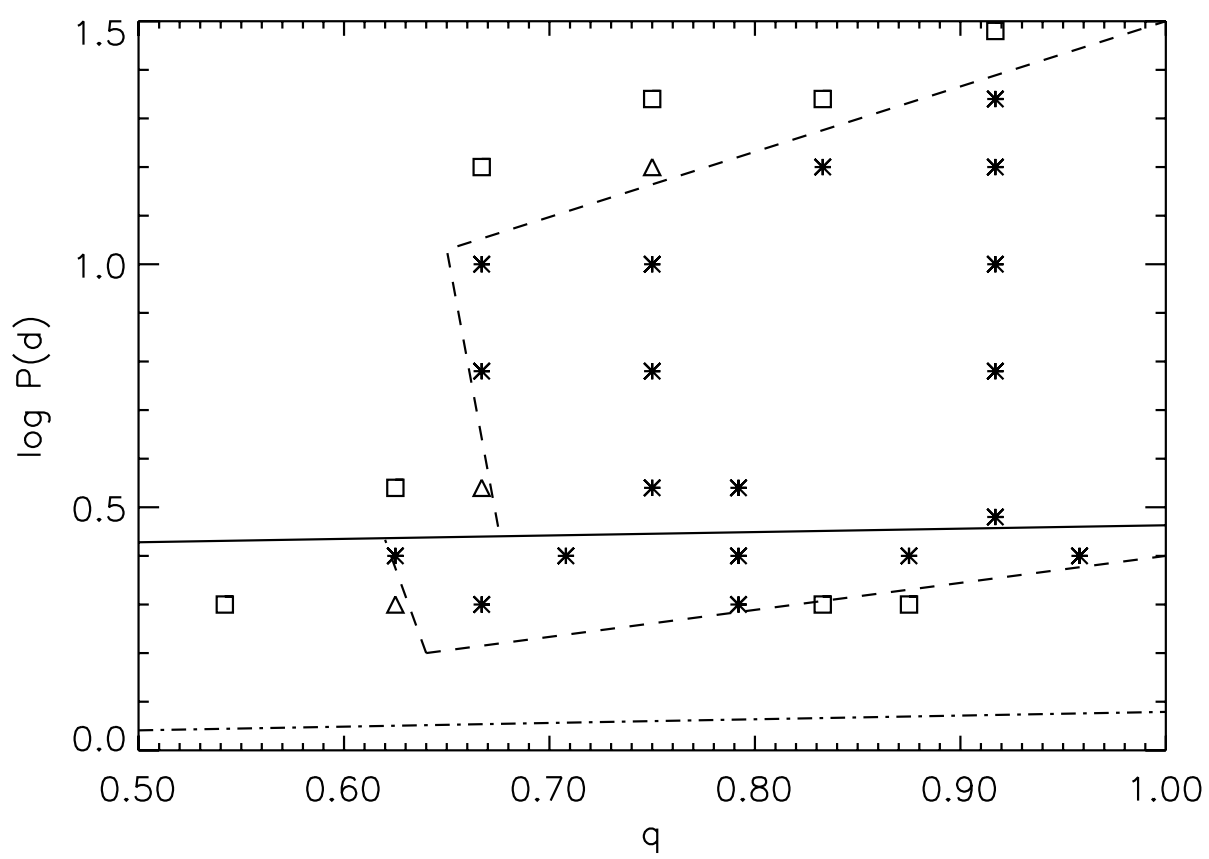

Figure 8. Distribution of all computed binaries with $12 \mathrm{M}_{\odot}$ primaries of Wellstein et al. (2001) in the initial period versus initial mass ratio diagram. Asterisks mark contact-free systems, while squares mark systems which evolve into contact. Systems marked with triangles are borderline cases, i.e., they evolve into a short contact phase but the secondary radius never exceeds its Roche radius by more than a factor 1.5. The solid line separates Case A (below) and Case B systems. All case A systems for this primary mass have a reverse supernova order. The dashed lines indicate the boundary between contact-free and contact evolution. The dashed-dotted line is defined by the condition that the primary fills its Roche lobe already on the zero age main sequence.

since thermohaline mixing dilutes the accreted matter with its whole envelope. Figure 9 gives an idea of the maximum obtainable enrichment, which, in the mass range considered here, amounts typically to 0.6 dex, where some Case A systems can produce as much as 0.8 dex. Non-conservative systems, where much of the overflowing matter is ejected from the binary system, are expected to obtain substantially smaller enrichments.

Secondly, nitrogen can be enhanced in mass gainers due to rotational mixing. Since these stars are amongst the most rapidly rotating main sequence stars, rotational mixing in these stars might be substantial. In fact the second panel in Fig. 6 shows that, while accretion has raised the nitrogen surface mass fraction by $\sim 0.4$ dex, it further-on slowly increases to a total enrichment of almost $\sim 0.7$ dex. Fig. 10 shows a more dramatic example: the same binary system as shown in Fig. 6 , but with an initial period of $6 \mathrm{~d}$ instead of $3 \mathrm{~d}$, accelerates its mass gainer to a rotational velocity of almost $500 \mathrm{~km} \mathrm{~s}^{-1}$, which gives an extra nitrogen enrichment of more than a factor two, to about 1 dex in total. At low metallicity, Cantiello et al. (2007) has found that the mass gainer of a $16 \mathrm{M}_{\odot}+15 \mathrm{M}_{\odot}$ system with an initial period of $5 \mathrm{~d}$ evolves chemically homogeneously after the Case B mass transfer event.

So we see that, quite naturally, close binaries produce rapidly rotating nitrogen-rich stars. This remains true if rotational mixing is completely neglected, where the nitrogen 


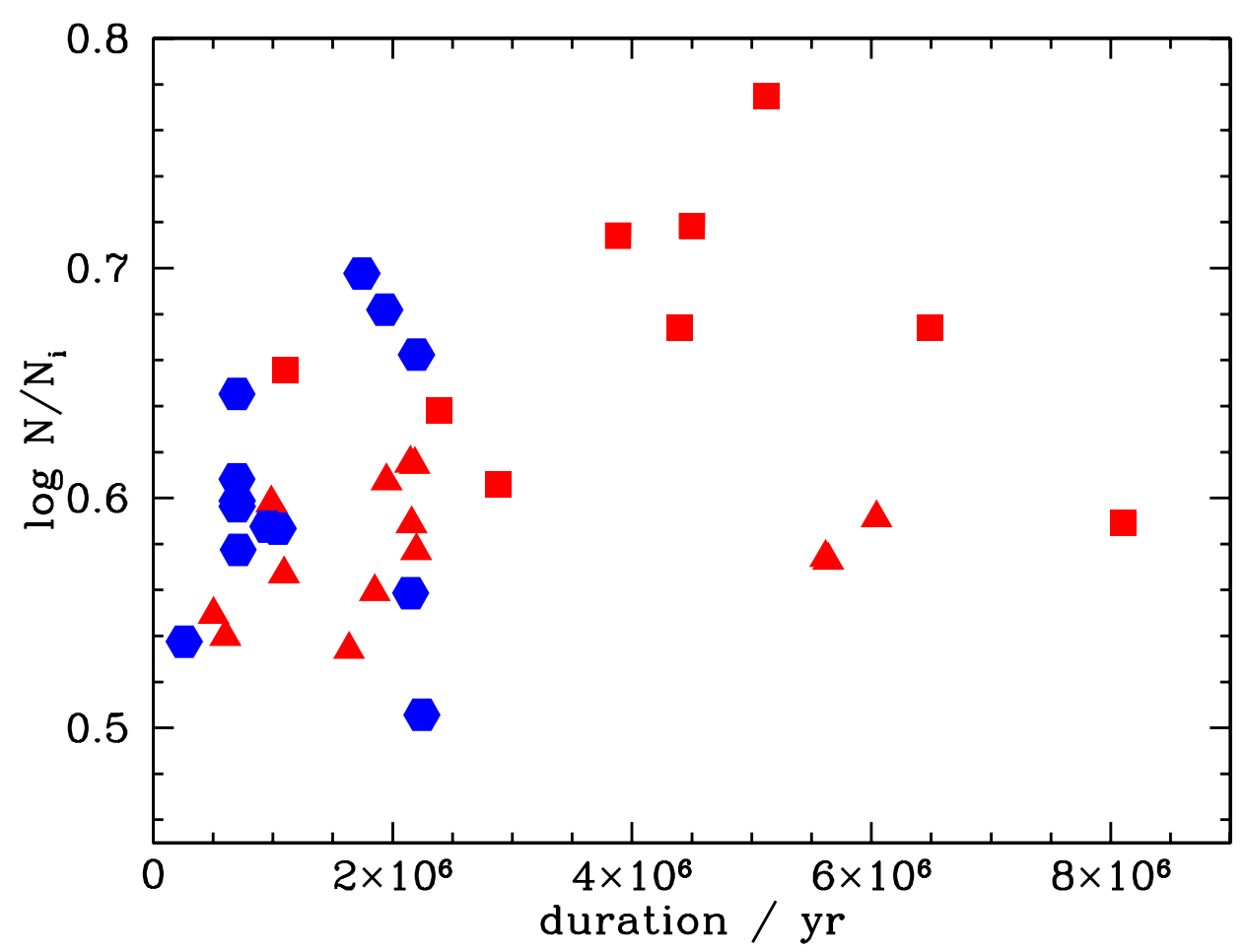

Figure 9. Surface abundance of nitrogen relative to its initial abundance for the solar metallicity binary models of Wellstein et al. (2001), as function of the duration of the post-mass transfer phases. The models are conservative, have $12 \mathrm{M}_{\odot}$ and $16 \mathrm{M}_{\odot}$ primaries, and initial mass ratios larger than 0.5. Case A mass gainers during slow Case A mass transfer (Algol-type systems) are marked by triangles. The same stars appear as squares after Case AB mass transfer. Post Case B mass gainers are marked by hexagons.

enhancement would just be a factor of $2 \ldots 3$. With rotational mixing included, a factor of 10 can be reached.

But Fig. 10 also shows that binaries can indeed produce rapid rotators with little enrichment (cf. lower two panels in Fig. 7). Note that the highly non-conservative evolution which is required to obtain this may be largely underrepresented in Fig. 10, as the corresponding binary evolution models are numerically difficult.

Finally, as the post-mass transfer period in very close systems can lead to rapid tidal spin-down (e.g., in the $2.15 \mathrm{~d}$ binary shown in Fig. 10), close binaries may also produce slowly rotating nitrogen-rich main sequence stars.

\section{Conclusions}

We have shown above that an effort is needed to compare the predictions of stellar evolution theory with the new results derived from the FLAMES Survey of Massive Stars (cf. Fig. 1). In particular, binary evolution models which include rotational mixing, mass and angular momentum transfer, and tidal interaction are required, of which only few exist today (cf., Fig. 10).

Fig. 10 can not be directly compared with Fig. 1. It does not contain a clean population study, and can therefore only indicate which parts of the diagram might be populated by binary systems, but not how many stars one might expect in those parts. Nevertheless, 


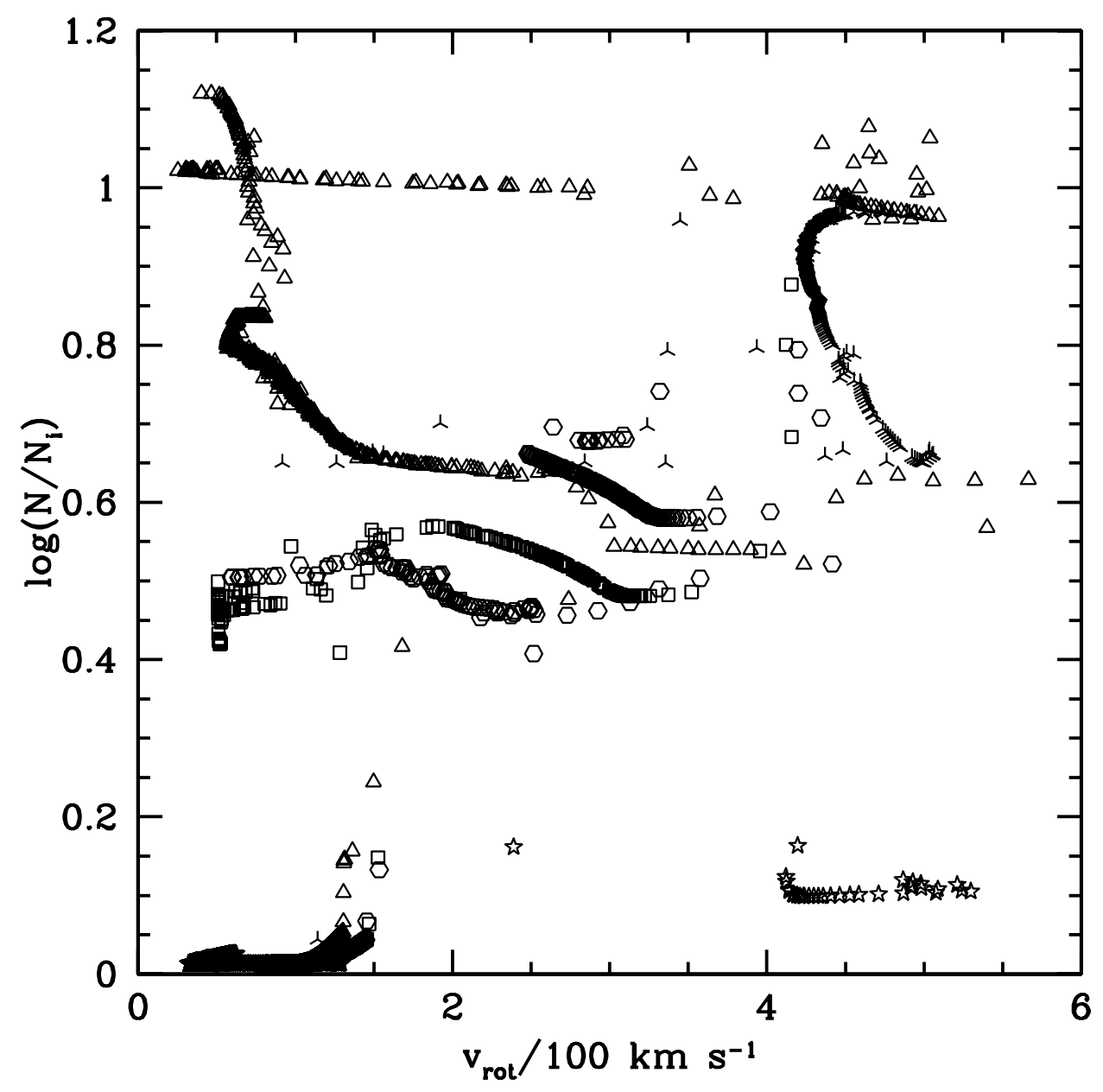

Figure 10. Nitrogen enhancement versus rotational velocity diagram, for the mass gainers of 5 computed binary systems, where each data point represents a duration of $20000 \mathrm{yr}$. All but one system start out with $16 \mathrm{M}_{\odot}+15 \mathrm{M}_{\odot}$. Open hexagons are used for the Case A system shown in Fig. 6, while open squares show the analogous system with a 10 time stronger tidal force parameter. Three-spiked stars designate a Case B system with an initial period of $6 \mathrm{~d}$, while five-spiked stars show the $8 \mathrm{~d}$ system from Fig. 7. Triangles mark a $10 \mathrm{M}_{\odot}+9 \mathrm{M}_{\odot}$ Case A system with an initial period of $2.15 \mathrm{~d}$. Only the core-hydrogen burning stage of the mass gainers is shown. As in Fig. 9, it can be seen that the enrichment by mass transfer alone goes up to about $\log N / N_{\mathrm{i}} \simeq 0.6$, with larger enrichments predominantly produced by rotational mixing.

it appears rather striking that the few models shown in Fig. 10 can populate each part of the diagram in which Fig. 1 shows a significant density of stars. In fact, one may ask the question whether single stars are needed at all to understand Fig. 1, except for the slowly rotating non-enriched stars.

This question ties in with the one asked in Sect. 4: Could all rapid rotators be spunup mass gainers and merger products? While it seems difficult to answer this question currently, the following statement seems secure: The FLAMES result shows that it is impossible to understand the nitrogen enhancement in massive stars without considering 
binary evolution. Whether the same statement can be made for single stars is questioned by the results shown above.

\section{Acknowledgements}

SCY was supported by the DOE Program for Scientific Discovery through Advanced Computing and NASA.

\section{References}

Berger, L., Koester, D., Napiwotzki, R., et al. 2005, A\& A, 444, 565

Belczynski, K., Kalogera, V., \& Bulik, T. 2002, ApJ, 572, 407

Bradley, P. A. 1998, ApJS, 116, 307

Bradley, P. A. 2001, ApJ, 552, 326

Braun, H. \& Langer, N. 1995, A\& A, 297, 483

Brinkworth, C. S., Burleigh, M. R., \& Marsh, T. R. 2007, in: R. Napiwotzki \& M. R. Burleigh (eds.), 15th European Workshop on White Dwarfs (San Francisco: ASP), ASP Conf. Ser., 372,183

Camilo, F., Ransom, S. M., Halpern, J. P., \& Reynolds, J. 2007, ApJ, 666, L93

Cantiello, M., Yoon, S.-C., Langer, N., \& Livio, M. 2007, A\&SA, 465, L29

Detmers, R., Langer, N., Podsiadlowski, P., \& Izzard, R. 2008, A\&AA, in press, astro$\mathrm{ph} / 0804.0014$

Dolez, N., Vauclair, G., Kleinman, S. J., et al. 2006, A\&A, 446, 237

Eldridge, J. J., Izzard, R. G., Tout, C. A. 2008, 384, 1109

Evans, C. J., Smartt, S. J., Lee, J.-K., et al. 2005, A\&SA, 437, 467

Ferrario, L. \& Wickramasinghe, D. T. 2005, MNRAS, 356, 615

Handler, G. 2001, MNRAS, 323, L43

Handler, G., Romero-Colmenero, E., \& Montgomery, M. H. 2002, MNRAS, 335, 399

Heger, A. \& Langer, N. 2000, ApJ, 544, 1016

Heger, A., Langer, N., \& Woosley, S. E. 2000, ApJ, 528, 368

Heger, A., Woosley, S. E., \& Spruit, H. C. 2005, ApJ, 626, 350

Hunter, I., Brott, I., Lennon, D. J., et al., 2008, ApJ, 676, L29

Kepler, S. O., Giovannini, O., Wood, M. A., et al. 1995, ApJ, 447, 874

Kleinman, S. J., Nather, R. E., Winget, D. E., et al. 1998, ApJ, 495, 424

Langer, N. 1998, A\&A, 329, 551

Maeder, A. \& Meynet, G. 2000, ARAA, 38, 143

Maeder, A. \& Meynet, G. 2005, A\&A, 440, 1041

Meynet, G. \& Maeder, A. 2000, $A \& A, 361,101$

Mokiem, M. R., de Koter, A., Evans, C. J., et al. 2006, A\&SA, 456, 1131

Packet, W. 1981, A\&A, 102, 17

Petrovic, J., Langer, N., Yoon, S.-C., \& Heger, A. 2005a, A\&A, 435, 247

Petrovic, J., Langer, N., \& van der Hucht, K. A. 2005b, A\&A, 435, 1013

Podsiadlowski, P., Joss, P. C., \& Hsu, J. J.L. 1992, ApJ, 391, 246

Pols, O. R. \& Marinus, M. 1994, A\&A, 288, 475

Spruit, H. C. 2002, A\&\&A, 381, 923

Suijs, M. P. L., Langer, N., Poelarends, A.-J., et al. 2008, A\& A, 481, L87

Vanbeveren, D., Van Bever, J., \& Belkus, H. 2007, ApJ, 662, L107

Vazquez, G. A., Leitherer, C., Schaerer, D., et al. 2007, ApJ, 663, 995

Wellstein, S. 2001, PhD thesis, University Potsdam

Wellstein, S. \& Langer, N. 1999, A\&A, 350, 148

Wellstein, S., Langer, N., \& Braun, H. 2001, A\& $A, 369,939$

Winget, D. E., Nather, R. E., \& Clemens, J. C. 1994, ApJ, 430, 839

Woosley, S. E. \& Heger, A. 2006, ApJ, 637, 914

Yoon, S.-C. \& Langer, N. 2005, A\&\&A, 443, 643

Yoon, S.-C., Langer, N., \& Norman, C. 2006, A\&A, 460, 199 